\title{
The influence of gait cadence on the ground reaction forces and plantar pressures during load carriage of young adults
}

\author{
Marcelo P. Castro; Maria Cristina Figueiredo; Sofia Abreu; Helena Sousa; Leandro \\ Machado; Rubim Santos; João Paulo Vilas-Boas
}

\begin{abstract}
Biomechanical gait parameters-ground reaction forces (GRFs) and plantar pressures-during load carriage of young adults were compared at a low gait cadence and a high gait cadence. Differences between load carriage and normal walking during both gait cadences were also assessed. A force plate and an in-shoe plantar pressure system were used to assess 60 adults while they were walking either normally (unloaded condition) or wearing a backpack (loaded condition) at low (70 steps per minute) and high gait cadences (120 steps per minute). GRF and plantar pressure peaks were scaled to body weight (or body weight plus backpack weight). With medium to high effect sizes we found greater anterior-posterior and vertical GRFs and greater plantar pressure peaks in the rearfoot, forefoot and hallux when the participants walked carrying a backpack at high gait cadences compared to walking at low gait cadences. Differences between loaded and unloaded conditions in both gait cadences were also observed.
\end{abstract}

\section{Keywords}

Backpack; Walking; Gait speed

\section{Introduction}

Standing or walking with backpacks shifts posterior and superiorly the combined centre of mass of the system backpack and backpacker, inducing postural imbalance for static and dynamic conditions (Singh and Koh, 2009). When wearing a backpack there is an increase in the load carried by the musculoskeletal system, which may lead to adaptation in postures and forces acting on the human body. Many studies indicated that load carriage changed the kinematics (Attwells et al., 2006, Birrell and Haslam, 2010, Birrell et al., 2007, Cobb and Claremont, 1995 and Majumdar et al., 2010), ground reaction forces (GRFs) (Birrell and Haslam, 2010, Birrell et al., 2007, Castro et al., 2013, Castro et al., 2014a, Cobb and Claremont, 1995 and Simpson et al., 2012), and plantar pressures (Castro et al., 2013 and Castro et al., 2014b) of walking. These biomechanical changes caused by load carriage might 
contribute to the high levels of back pain (Grimmer and Williams, 2000 and Skaggs et al., 2006), muscle discomfort (Johnson et al., 1995), joint problems (Birrell and Haslam, 2009), metatarsal stress fractures (Hodge et al., 1999), metatarsalgia (Knapik et al., 1992 and Pau et al., 2011), and foot blisters (Knapik et al., 1996) observed in people wearing backpacks.

Changing gait speed or cadence-the relationship between cadence and speed during walking tends to be linear (Perry, 1992)_influences many biomechanical gait parameters during "normal" walking (without a backpack). At higher gait speeds, the vertical and anterior-posterior GRFs (Chiu and Wang, 2007, Chung and Wang, 2010 and Goble et al., 2003), and the plantar pressure peaks in the heel and medial forefoot were higher, whereas in the midfoot and lateral forefoot they were lower (Rosenbaum et al., 1994) compared to slower gait speeds. Previous studies observed changes in gait stability (Hsiang and Chang, 2002), and in spatial-temporal gait parameters (Charteris, 1998) as a consequence of changing gait speed during load carriage. However, it is unclear the influence of gait speed on kinetic gait parameters, such as shear and vertical forces and plantar pressures, during load carriage.

The GRFs and plantar pressure approaches are insightful techniques for assessing gait biomechanics. The vertical GRF provide information about mechanical stress (Piscoya et al., 2005). This measure might be related to joint contact forces, which appear to play an important role in the development of pathological conditions such as low back pain and osteoarthritis (Piscoya et al., 2005). The anterior-posterior GRF is the main component that indicates shear stress (Chang et al., 2011). This force provides insights into the friction between the foot and shoe or shoe and ground, and their increase might be linked to the development of foot-related injuries such as foot blisters (Knapik et al., 1992), and tendency to slip (Chang et al., 2011). Both of the mentioned GRF components inform about the overall forces acting on the human body. However, the GRFs do not provide any information about where the forces are being applied on the foot (Castro et al., 2014c). In-shoe plantar pressure systems allow quantification of the amount of vertical GRF being applied on each region of the plantar surface, providing information about foot structure and function (Cavanagh and Ulbrecht, 1994). Positive correlation between plantar pressure peaks and pain ratings have been previously found (Hodge et al., 1999). Finally, to achieve a more detailed and comprehensive picture of the forces acting on the musculoskeletal system, combining the GRFs and plantar pressure analyses appears to be relevant.

Students, hikers and soldiers often change their gait cadence while they are walking, or, depending on their aims, adopt a low or high gait cadence during 
activity. The knowledge of the loads imposed to the musculoskeletal system caused by alterations in gait cadence may help in developing accessories (e.g. shoes and insoles) more suitable for specific gait conditions (walking with or without a backpack, at a low or high gait cadence), as well as to identify plantar foot areas more susceptible to damage, with the purpose of making the activity safer and more comfortable and preventing injuries. Therefore, the aim of this study was to compare biomechanical gait parameters-GRFs and plantar pressures_during load carriage of young adults at a low gait cadence and a high gait cadence. We also assessed differences on these biomechanical gait parameters between load carriage and normal walking during both gait cadences. We hypothesised that during load carriage at the high gait cadence higher GRFs and plantar pressures will be found compared to the low gait cadence. We also hypothesised that differences in GRFs and plantar pressures will be observed between load carriage and normal walking (values scaled to body weight plus backpack weight for the load carriage condition, and scaled to body weight for the normal walking condition) in both gait cadences.

\section{Methods}

\subsection{Participants}

All participants were physically active and their body mass indexes (BMIs) were between 18 and $25 \mathrm{~kg} / \mathrm{m}^{2}$. They were excluded if they presented any traumaticorthopaedic dysfunction or difficulties on independent walking. Sixty participants (30 males and 30 females) with age of $22.8 \pm 3.8$ years old, weight of $65.5 \pm 9.8 \mathrm{~kg}$, height of $168.8 \pm 8.8 \mathrm{~cm}$, and BMI of $22.8 \pm 1.7 \mathrm{~kg} / \mathrm{m}^{2}$ were enrolled in this investigation. This experimental repeated-measures study was approved by the local ethical committee and all participants freely signed an informed consent term, based on Helsinki's declaration, which explained the purpose and the procedures of the study.

\subsection{Apparatus}

To record the GRFs, we used a Bertec force plate model 4060-15 (Bertec Corporation, Columbus, USA), operating at $1000 \mathrm{~Hz}$. To assess the plantar pressure distribution, we used an F-Scan insole pressure system (TekScan, South Boston, USA), operating at $300 \mathrm{~Hz}$ with about 960 pressure cells (depending on the foot size) and a $0.18 \mathrm{~mm}$ thick insole sensor. We used a metronome (Wittner Maelzel Metronome, Germany) to control gait cadence, and walking speed was measured by videogrammetry using three digital video camera recorders. An external trigger was developed to synchronise the force 
plate, in-shoe plantar pressure system, and video by starting them simultaneously.

\subsection{Tasks and procedures}

The participants underwent three phases at the lab: preparation, familiarisation and test. In the first phase, the study procedures were explained to the participants, and their weight and height were recorded using the force plate and a stadiometer (Seca, Birmingham, United Kingdom), respectively. For each participant, the external weight required to raise their total weight (body weight plus backpack weight) to a "loaded BMl" of $30 \mathrm{~kg} / \mathrm{m}^{2}$ was calculated (Castro et al., 2013). Then a backpack was filled with sand and fixed at the central area of each participant's back (Fig. 1). The participants were allowed adjusting the position of the backpack to make it the most similar with the position they usually used. We selected this backpack model as it was the most used among the participants in preliminary studies performed by our research group. There is no well-established recommendation of backpack's weight limit for young adult population based on biomechanical parameters. Obesity (BMI $>30)$ is associated with elevated risk of both degenerative and inflammatory musculoskeletal conditions (King et al., 2013). Thus, even recognizing differences in weight distribution between obese people and subjects using a backpack, we used the total amount of mechanical load found in obese individuals as criterion in order to assess the human locomotor system during a challenging condition. So, the weight placed inside the backpack was $20.3 \pm 4.4 \mathrm{~kg}$, allowing an "artificial" BMI of 30 for each subject. 


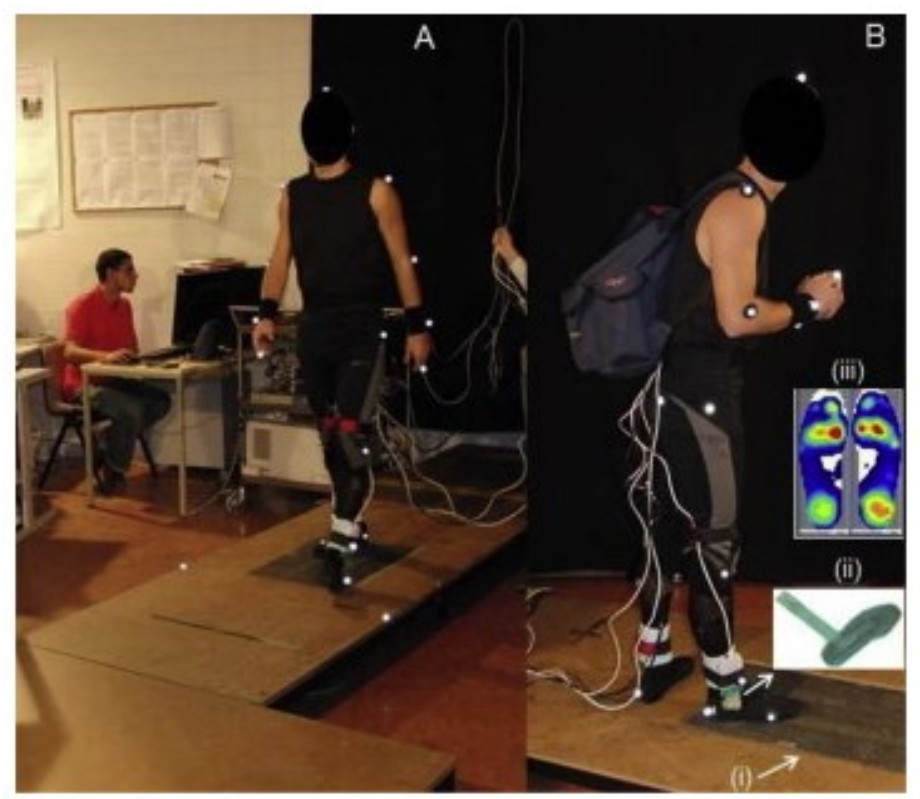

Fig. 1.

Experimental setup (A); and participant setup (B). Force plate (i), sensor insole (ii) and plantar pressure data from one trial (iii).

A cuff unit measuring $98 \times 64 \times 29 \mathrm{~mm}$ was attached with Velcro straps up the lateral malleolus region of both legs of the participants and a $9.25 \mathrm{~mm}$ cable linked the cuff to the VersaTek hub (F-Scan system), which was beside the walkway connected to a computer; the cable did not cause any restriction for walking (Fig. 1). A pair of thin socks and, aiming to minimise the effects of different soles, a neutral shoe (ballet sneaker) with the sensor insoles inside, were provided for all participants. Twenty one reflective markers with a diameter of $1.2 \mathrm{~cm}$ were placed with adhesive tape on the participants. Only data from the marker at the right great trochanter of the femurs was analysed in the current study.

Afterwards, subjects were allowed to become familiar with walking over a walkway at 70 steps per minute (labelled as low gait cadence) and 120 steps per minute (labelled as high gait cadence) paced using the metronome. The force plate was embedded in the middle of the walkway. At this moment, we identified the site where the participants should begin walking to step with their right foot at the centre of the force plate without altering their gait pattern. In the last phase, the participants performed six valid trials (three at low gait cadence and three at high gait cadence) without the backpack (labelled as unloaded condition) and six valid trails wearing the backpack (labelled as loaded condition). The order of the trials with the low and high gait cadences was counterbalanced. The participants performed two steps before and after reaching the force plate (3-step protocol), therefore avoiding the effects of acceleration (Macfarlane and Looney, 2008). 


\subsection{Data analysis}

For the acquisition of the force plate and in-shoe pressure system data the Acknowledge software (BIOPAC System ${ }^{\mathrm{TM}}$, California, USA) and the software F-Scan Research 6.33 (TekScan ${ }^{\mathrm{TM}}$, South Boston, USA), were respectively used. Data were exported to Matlab 7.0 software (MathWorks ${ }^{\mathrm{TM}}$, Massachusetts, USA) and a program was specifically developed for the processing and calculations of the dependent variables.

We calculated four dependent variables based on the GRF records: the load acceptance peak (Fv1 -the first peak of the vertical GRF); the thrust peak (Fv2 - the second peak of the vertical GRF); the braking peak (Fap1-the first "negative" peak of the anterior-posterior GRF); and the propulsive peak (Fap2the second "positive" peak of the anterior-posterior GRF).

Regarding the in-shoe pressure system data, the program automatically divided the footprint into 10 plantar foot regions, as previously proposed (Castro et al., 2013 and Cavanagh and Ulbrecht, 1994): hallux, distal phalanges, medial, central and lateral forefoot; medial and lateral midfoot; medial, central, and lateral rearfoot. Nevertheless, this procedure was always verified by visual inspection, and eventually, the boundaries between foot regions were manually corrected. For each of the 10 plantar foot regions, the program calculated its corresponding plantar pressure peak, which was considered the highest pressure value shown on a sensor during the stance phase when the participant stepped on the force plate. The force plate was used to calibrate the plantar pressure data as previously described (Castro et al., 2013).

Dvideo v.5.0 system (Unicamp, Campinas, Brazil) (Figueroa et al., 2003) was used to capture, synchronise, digitalise and reconstruct the images for $3 \mathrm{D}$ kinematical analysis. The walking speed was calculated by the first time derivative of the great trochanter reflective marker of the horizontal anteriorposterior position. The walking speed was used as a control variable to verify whether the participant during the unloaded and loaded conditions walked at the same speed at the low and high gait cadences.

Both GRF and plantar pressure data were scaled to the total weight (TW) of the condition: for the unloaded condition they were scaled to the participants' body weight, whereas for the loaded condition they were scaled to the participants' body weight plus backpack weight. The GRF ( $\left.{ }^{*} T W / N\right)$ was expressed as TW unit, and the plantar pressures $\left(\mathrm{N}^{*} \mathrm{TW} / \mathrm{N} / \mathrm{cm}^{2}\right)$ was expressed as $\mathrm{TW}$ unit per square centimetre $\left(\mathrm{TW} / \mathrm{cm}^{2}\right)$. 


\subsection{Statistical analysis}

Statistical analysis was performed using the Statistica ${ }^{\circledR}$ v.8 software (StatSoft $®$, Tulsa, USA) with an a value set at 0.05 . Intra-class correlation coefficient (ICC) was calculated to verify the inter-trial repeatability of the dependent variables (GRF and plantar pressure peaks). Then the mean value of each of the participants' three repetitions was calculated for each dependent variable and used to perform all statistical tests as representative of the subject's response. Two repeated measures MANOVAs were conducted to analyse differences between low and high gait cadences, and between unloaded and loaded conditions on the: (i) GRF peaks and (ii) plantar pressure peaks. In both repeated measures MANOVAs the gait cadences (low and high), and load conditions (unloaded and loaded) were considered as within-subject factors, and the: (i) GRF peaks, and (ii) plantar pressure peaks as dependent measures. One repeated measure ANOVA was used to compare the walking speed between load conditions during the low and high gait cadences. Whenever a statistically significant difference was found, the Fisher's Least Significant Difference was calculated. Considering MANOVA assumptions, the data were found to be normal as indicated by the Kolmogorov-Smirnov test $(p>0.05)$, and the sphericity verified by the Mauchly's test, was held. We used the partial Eta square $\left(\eta^{2}\right)$ to measure the effect sizes considering that an $\eta^{2}$ of 0.01 or less was small, of 0.06 was medium, and of 0.14 or more was large (Stevens, 2002).

\section{Results}

Good to excellent inter-trial repeatability was found for all variables (ICC > 0.80). No statistical differences in walking speed were observed between unloaded $(0.77 \pm 0.07 \mathrm{~m} / \mathrm{s})$ and loaded conditions $(0.76 \pm 0.06 \mathrm{~m} / \mathrm{s})$ at the low gait cadence $(p=0.21)$. During the high gait cadence the participants walked faster $(p=0.01)$ during the loaded condition $(1.34 \pm 0.11 \mathrm{~m} / \mathrm{s})$ compared to the unloaded condition $(1.29 \pm 0.11 \mathrm{~m} / \mathrm{s})$.

Interactions were observed between load conditions and gait cadences in the GRF peaks $\left(F(3,177)=10.34, p<0.001, \eta^{2}=0.15\right.$, power $=99.8 \%-$ Fig. 2$)$. During load carriage, all GRF peaks were higher $(p<0.001)$ in the high gait cadence compared to the low gait cadence. The differences between gait cadences ranged from 0.059 TW in the Fap2 to 0.081 TW in the Fv1. In the low gait cadence, similar values between unloaded and loaded conditions were found in Fv1, Fv2 and Fap2 ( $p>0.05)$, whilst Fap1 was lower $(p<0.001)$ during loaded compared to unloaded condition (Fig. 2). Considering the high gait 
cadence, higher values were observed in the vertical GRF (Fv1 and Fv2) during load carriage compared to the unloaded condition (Fig. 2A, $p<0.001$ ), whilst the anterior-posterior GRF (Fap1 and Fap2) were similar between conditions (Fig. 2B, $\mathrm{p}>0.05$ ). The greater differences between unloaded and loaded conditions were observed in the Fv1 (0.031 TW) and Fv2 (0.041 TW) and during the high gait cadence.
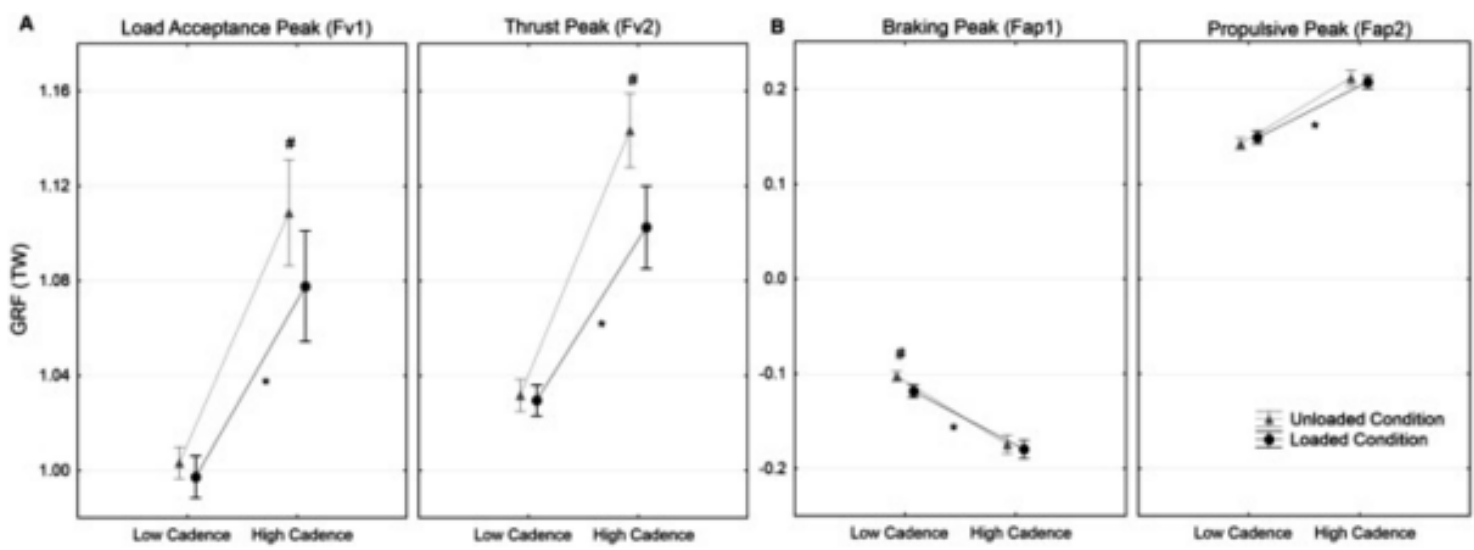

Fig. 2 .

Interactions between load conditions and gait cadences in the ground reactions forces (GRFs): (A) vertic al GRF, (B) anterior-posterior GRF. TW = total weight (the same than the body weight for the unloaded condition, and the body weight plus the backpack weight for the loaded condition). ${ }^{\star}=$ statistic ally signific ant differences between gait cadences with $p<0.05$; \# = statistic ally signific ant differences between unloaded and loaded conditions with $p<0.05$. The triangles and circles represent the average, and the error bars the $95 \%$ confidence interval.

Interactions were found between load conditions and gait cadences in the plantar pressure peaks $\left(F(9,522)=3.63, p<0.001, \eta^{2}=0.06\right.$, power $=99.2 \%$ - Fig. 3). During load carriage at the high gait cadence, higher plantar pressure peaks were observed in the hallux, forefoot and rearfoot (medial, central and lateral) regions compared to the low gait cadence. The opposite was found in the lateral midfoot (higher values at the low gait cadence), and similar values between both gait cadences in the distal phalanges and medial midfoot were observed (Fig. 3). The greater differences in plantar pressure peaks between gait cadences during load carriage were observed in the medial and central rearfoot regions (Fig. 3). 


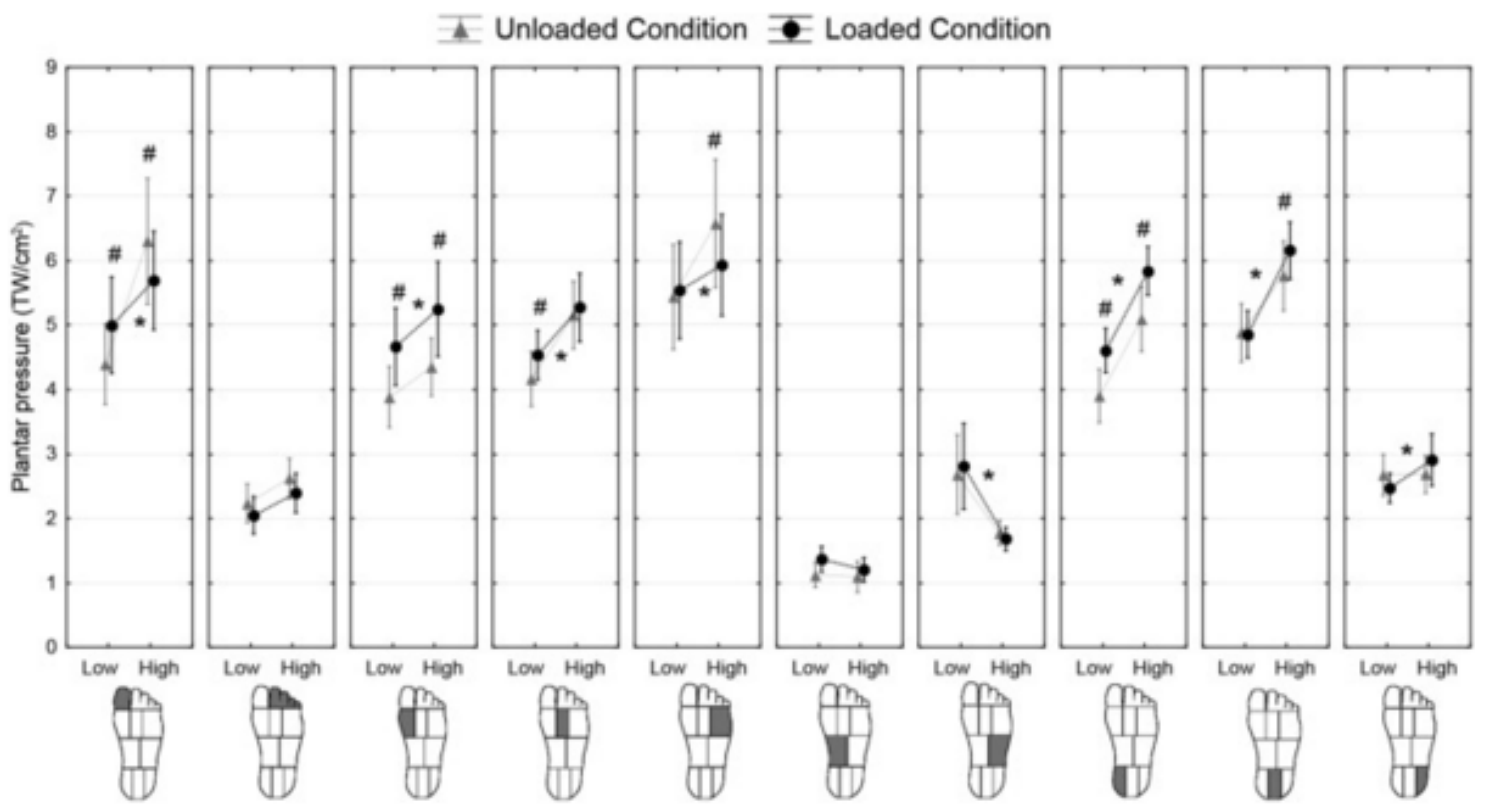

Fig. 3 .

Interactions between load conditions and gait cadences in the plantar pressure peaks. TW = total weight (the same than the body weight for the unloaded condition, and the body weight plus the backpack weight for the loaded condition). ${ }^{*}=$ statistic ally signific ant differences between gait cadences with $p<0.05$; \#=statistic ally signific ant differences between unloaded and loaded conditions with $p<0.05$. The triangles and circles represent the average, and the error bars the $95 \%$ confidence interval.

When unloaded and loaded conditions were compared at the low gait cadence, we found greater plantar pressure peaks in the hallux $(p=0.001)$, medial $(p<0.001)$ and central $(p=0.047)$ forefoot and medial rearfoot $(p<0.001)$ during load carriage, whilst in the other regions similar values were found between load conditions ( $p>0.05$ ). During the high gait cadence, in the loaded condition we found greater plantar pressure peaks in the medial forefoot $(p<0.001)$, medial $(p<0.001)$ and central rearfoot $(p=0.04)$ compared to the low gait cadence; however, in the hallux $(p=0.001)$ and lateral forefoot $(p<0.001)$ lower values were observed in the loaded compared to the unloaded condition (Fig. 3). The greater differences in plantar pressure peaks between unloaded and loaded conditions were observed in the medial forefoot and medial rearfoot, where higher values occurred during load carriage (Fig. 3).

\section{Discussion}

The purpose of the current study was to verify differences on GRFs and plantar pressures while walking carrying a load between low and high gait cadences. We also compared these biomechanical gait parameters between load carriage and normal walking at both gait cadences. We scaled the data to the total load (body weight for the unload condition, and body weight plus backpack for the 
loaded condition) to assess changes in GRF and plantar pressures not directly related to inertial effects of increased load. The first hypothesis of this study was partially satisfied as we did find higher GRF peaks (for Fap1 in module) and in seven out of the 10 foot regions during load carriage at the high gait cadence compared to the low gait cadence; however, no differences between gait cadences were observed in the distal phalanges and medial midfoot, and higher plantar pressure peaks occurred in the lateral midfoot during the low gait cadence. Our second hypothesis, which suggested differences in GRFs and plantar pressures between unloaded and loaded conditions, was satisfied. We observed in both GRF and plantar pressure peaks substantial differences between load conditions. The differences in biomechanical gait parameters found between gait cadences, and between load conditions were not only statistically significant, but they also appear to be relevant in practice as medium to high effect sizes were observed.

Higher GRF peaks were found in the high compared to the low gait cadence while walking carrying a backpack. These effects might be explained through the increased acceleration characteristic of high gait cadences. The heel strike phase (Fv1 and Fap1) is when the body segmental speeds require the highest inertial forces to decelerate the human body and backpack masses (Knapik et al., 1998). While during the propulsion phase of the stance (Fv2 and Fap2), a more intense force is needed to accelerate the system (Knapik et al., 1998). These expected increases in GRF peaks in higher gait cadences have already been described while people walked without a backpack (Chiu and Wang, 2007, Chung and Wang, 2010, Orendurff et al., 2008 and Voloshin, 2000). Therefore, at higher gait cadences greater magnitudes of forces are generated at the heel strike, imposing, as a consequence, higher demands to the musculoskeletal system (Voloshin, 2000). Complementarily, joint contact forces will also be increased (Piscoya et al., 2005), particularly if muscles activity can't cope with the increased load.

Interestingly, a reduction in the vertical GRF (Fv1 and Fv2) was observed during walking at a high gait cadence while carrying a backpack compared to the unloaded condition (Fig. 2a). This behaviour suggests a protective gait pattern adaptation as a consequence of load carriage, aiming to minimise possible harmful effects of high vertical GRF over the musculoskeletal system. The same protective gait pattern during load carriage has already been described at a self-selected walking speed (Castro et al., 2013 and Simpson et al., 2012). However, during the low gait cadence no changes in the vertical GRF were observed. These findings suggest changes in gait strategy at either 
self-selected or high gait cadences during load carriage. It might occur to protect the locomotor system from risk associated to excessive vertical forces. Considering the shear forces, during the low gait cadence we observed higher magnitudes of braking forces (Fap1) during load carriage compared to the unloaded condition, whereas in the high gait cadence similar values were observed between loaded and unloaded conditions (Fig. 2b). These findings suggest that the shear stress increased more than the proportion of the backpack weight did in low gait cadences. Another study assessed the influence of load carriage while the participants were walking at a self-selected pace, corroborates with these findings (Castro et al., 2013). Thus, there are changes in the gait pattern during loaded conditions - compared to unloaded walking - at self-selected and slow gait cadences that possibly determines a higher susceptibility to plantar foot-related injuries (Knapik et al., 1992) and of slipping (Chang et al., 2011) when an extra load is carried.

Some studies evaluated the influence of load carriage (Castro et al., 2013) and gait speed (Judith et al., 2004, Rosenbaum et al., 1994 and Segal, 2004) on the plantar pressure parameters during walking. However, none of them analysed how the plantar pressures are affected by changing gait speed or cadence during load carriage. Therefore, we could not make any direct comparison to our findings. We observed during the loaded condition when the participants walked with a high gait cadence greater peaks in the rearfoot (medial and central), forefoot (medial, central and lateral) and hallux than at low gait cadence. On the other hand, lower plantar pressure peaks in the lateral midfoot during load carriage at higher gait cadences were observed. The greater differences occurred in the medial forefoot and medial rearfoot. Thus, since there is a positive correlation between plantar pressure peaks and pain ratings (Hodge et al., 1999), special care should be taken on these regions as the foot may not be used to and then do not know how to cope with those high magnitudes of pressure.

Authors that analysed the normal walking (unloaded condition) also found different plantar pressure peaks when the gait cadence changed (Judith et al., 2004, Rosenbaum et al., 1994 and Segal, 2004). At high gait cadences increased plantar pressure peaks in the rearfoot, medial forefoot (Judith et al., 2004, Rosenbaum et al., 1994 and Segal, 2004), central forefoot and hallux (Judith et al., 2004 and Segal, 2004), and lower plantar pressure peaks in the midfoot (which was considered as one region) and lateral forefoot (Rosenbaum et al., 1994) have been described. Overall, the findings of the current study are in agreement with the mentioned ones (Judith et al., 2004, Rosenbaum et al., 1994 and Segal, 2004). However, there were a few differences observed 
between our study and the aforementioned ones as we divided the foot into more regions. Therefore, we did find higher pressure peaks in the heel, however only in the medial and central regions. Regarding the decreased pressure peaks in the midfoot at high gait cadences (Rosenbaum et al., 1994), we support it only for the lateral region.

A better comprehension about the load applied to the human body during either professional activities or leisure is important to improve performance and safety of the activity. Our data suggest caution for those walking carrying a backpack at high gait cadences. We observed higher vertical and shear forces, and higher plantar pressure peaks in seven foot regions. Therefore, during load carriage individuals may be more susceptible to foot-related and limb injuries. Special attention should be paid in the medial rearfoot and medial forefoot as during load carriage changes in gait pattern-compared to normal walking-promoted a higher recruitment of these regions. Accessories such as shoes or insoles aiming to re-distribute the plantar pressures and promoting cushioning, could be developed for people while carrying a load. We recommend the relief of the medial surface of the foot by increasing recruitment of the midfoot and lateral foot regions. Considering prevention of musculoskeletal injury, we suggest to those people feeling any discomfort either in the lower limbs' joints or on the plantar surface to avoid walking at high gait cadences carrying a backpack.

There are some limitations in the present study. First, the backpack weight was not the same among the participants. However, we scaled the data to the participants' total weight (body weight plus backpack weight) and then we believe it did not have relevant influence in the results. Furthermore, we used the load criterion BMI $=30$ because there is no well-established load limit recommendation based on biomechanical parameters. Studies assessing physiological parameters proposed load limits of either between $30 \%$ and $33 \%$ of the body weight (Haisman, 1988, Simpson et al., 2011a and Simpson et al., 2011b) or ranging between 22.3 and $36.1 \mathrm{~kg}$ (Haisman, 1988, Knapik, 1989 and Pal et al., 2009). In the present study the $95 \%$ confidence interval of the applied load was $29.5-35.8 \%$ of body weight. We believe this load criterion was proper to assess the musculoskeletal system during a challenging condition. Second, the walking speed during the high gait cadence was statistically different between the unloaded $(1.29 \pm 0.11 \mathrm{~m} / \mathrm{s})$ and loaded condition $(1.34 \pm 0.11 \mathrm{~m} / \mathrm{s})$. Thus, we could expect higher values during load carriage as they walked faster than the unloaded condition. However, in general, the opposite occurred (lower values in the loaded condition). Thus we believe those small differences in gait speed barely influenced the results when both load conditions were compared at the high gait cadence. Furthermore, we 
chose to control gait cadence because a metronome can be easily used outside the lab, by an application in a mobile phone or in music players, and then it enhances the external validity of the study. Finally, we only assessed the right foot; therefore, we did not control the influence of limb dominance on data. However, we think that the assumption of gait symmetry (Goble et al., 2003), and similar GRFs observed between lower limbs in previous study (Seeley et al., 2008) minimises this limitation.

\section{Conclusion}

When walking carrying a backpack at high gait cadences the musculoskeletal system receives greater anterior-posterior and vertical GRFs and greater plantar pressure peaks in the rearfoot, forefoot and hallux compared to walking at low gait cadences. Comparing the loaded and unloaded conditions, we observed that load carriage influenced the magnitude and pattern that the forces were distributed on the plantar surface during walking. During loaded carriage, compared to normal walking, at low gait cadence the hallux, medial and central forefoot, and medial rearfoot were loaded more than the proportion of the load, and more shear stress during the first half of the stance phase was observed. In the high gait cadence, during load carriage we continued observing higher plantar pressure peaks in the medial forefoot and medial rearfoot compared to the unloaded condition. However, the hallux and lateral forefoot appears to be used less, as well as lower vertical GRF peaks were also observed.

\section{References}

Attwells et al., 2006

R.L. Attwells, S.A. Birrell, R.H. Hooper, N.J. Mansfield

Influence of carrying heavy loads on soldiers' posture, movements and gait

Ergonomics, 49 (2006), pp. 1527-1537

Birrell and Haslam, 2009

S.A. Birrell, R.A. Haslam

The effect of military load carriage on 3-D lower limb kinematics and spatiotemporal parameters

Ergonomics, 52 (2009), pp. 1298-1304 
Birrell and Haslam, 2010

S.A. Birrell, R.A. Haslam

The effect of load distribution within military load carriage systems on the kinetics of human gait

Appl. Ergon., 41 (2010), pp. 585-590

Birrell et al., 2007

S.A. Birrell, R.H. Hooper, R.A. Haslam

The effect of military load carriage on ground reaction forces

Gait Posture, 26 (2007), pp. 611-614

Castro et al., 2013

M. Castro, S. Abreu, H. Sousa, L. Machado, R. Santos, J.P. Vilas-Boas

Ground reaction forces and plantar pressure distribution during occasional loaded gait

Appl. Ergon., 44 (2013), pp. 503-509

Castro et al., 2014a

M.P. Castro, S. Abreu, V. Pinto, R. Santos, L. Machado, M. Vaz, J.P. Vilas-Boas

Influence of pressure relief insoles developed for loaded gait (backpackers and obese people) on plantar pressure distribution and ground reaction forces

Appl. Ergon., 45 (2014), pp. 1028-1034

Castro et al., 2014b

M.P. Castro, S.A. Abreu, H. Sousa, L. Machado, R. Santos, J.P. Vilas-Boas

In-shoe plantar pressures and ground reaction forces during overweight adults' overground walking

Res. Q. Exerc. Sport, 85 (2014), pp. 188-197

Castro et al., 2014c

M.P. Castro, M. Meucci, D.P. Soares, P. Fonseca, M. Borgonovo-Santos, F. Sousa,

L. Machado, J.P. Vilas-Boas

Accuracy and repeatability of the gait analysis by the WalkinSense system 
Biomed. Res. Int., 2014 (2014), p. 11

Cavanagh and Ulbrecht, 1994

P.R. Cavanagh, J.S. Ulbrecht

Clinical plantar pressure measurement in diabetes: rationale and methodology

Foot, 4 (1994), pp. 123-135

Chang et al., 2011

W.-R. Chang, C.-C. Chang, S. Matz

The effect of transverse shear force on the required coefficient of friction for level walking. Hum

Factors, 53 (2011), pp. 461-473

Charteris, 1998

J. Charteris

Comparison of the effects of backpack loading and of walking speed on footfloor contact patterns

Ergonomics, 41 (1998), pp. 1792-1809

Chiu and Wang, 2007

M.-C. Chiu, M.-J. Wang

The effect of gait speed and gender on perceived exertion, muscle activity, joint motion of lower extremity, ground reaction force and heart rate during normal walking

Gait Posture, 25 (2007), pp. 385-392

Chung and Wang, 2010

M.-J. Chung, M.-J.J. Wang

The change of gait parameters during walking at different percentage of preferred walking speed for healthy adults aged 20-60 years

Gait Posture, 31 (2010), pp. 131-135

Cobb and Claremont, 1995

J. Cobb, D. Claremont 
Transducers for foot pressure measurement: survey of recent developments Med. Biol. Eng. Comput., 33 (1995), pp. 525-532

Figueroa et al., 2003

P.J. Figueroa, N.J. Leite, R.M. Barros

A flexible software for tracking of markers used in human motion analysis

Comput. Methods Programs Biomed., 72 (2003), pp. 155-165

Goble et al., 2003

D.J. Goble, G.W. Marino, J.R. Potvin

The influence of horizontal velocity on interlimb symmetry in normal walking Hum. Mov. Sci., 22 (2003), pp. 271-283

Grimmer and Williams, 2000

K. Grimmer, M. Williams

Gender-age environmental associates of adolescent low back pain Appl. Ergon., 31 (2000), pp. 343-360

Haisman, 1988

M.F. Haisman

Determinants of load carrying ability

Appl. Ergon., 19 (1988), pp. 111-121

Hodge et al., 1999

M.C. Hodge, T.M. Bach, G.M. Carter

Orthotic management of plantar pressure and pain in rheumatoid arthritis

Clin. Biomech. (Bristol, Avon), 14 (1999), pp. 567-575

Hsiang and Chang, 2002

S.M. Hsiang, C. Chang

The effect of gait speed and load carrying on the reliability of ground reaction forces

Saf. Sci., 40 (2002), pp. 639-657 
Johnson et al., 1995

R. Johnson, J. Knapik, D. Merullo

Symptoms during load carrying: effects of mass and load distribution during a $20-\mathrm{km}$ road march

Percept. Mot. Ski., 81 (1995), pp. 331-338

Judith et al., 2004

M.B. Judith, D.F. Courtney, S.M. Olfat, P. Jacquelin

The influence of walking speed and footwear on plantar pressures in older adults

Clin. Biomech. (Bristol, Avon), 19 (2004), pp. 78-84

King et al., 2013

L.K. King, L. March, A. Anandacoomarasamy

Obesity \& osteoarthritis

Indian J. Med. Res., 138 (2013), pp. 185-193

Knapik, 1989

J. Knapik

Load Carried by Soldiers: Historical, Physiological, Biomechanical and Medical Aspects

U.S. Army Research Institute of Environmental Medicine, Natick, Masachusetts (1989) Report No T 19-89

Knapik et al., 1996

J.J. Knapik, E. Harman, K. Reynolds

Load carriage using packs: a review of physiological, biomechanical and medical aspects

Appl. Ergon., 27 (1996), pp. 207-216

Knapik et al., 1998

J.J. Knapik, K. Reynolds, J. Barson

Influence of an antiperspirant on foot blister incidence during cross-country hiking

J. Am. Acad. Dermatol., 39 (1998), pp. 202-206 
Knapik et al., 1992

J.J. Knapik, K. Reynolds, J. Staab, J. Vogel, B. Jones

Injuries associated with strenuous road marching

Mil. Med., 157 (1992), pp. 64-67

Macfarlane and Looney, 2008

P.A. Macfarlane, M.A. Looney

Walkway length determination for steady state walking in young and older adults

Res. Q. Exerc. Sport, 79 (2008), pp. 261-267

Majumdar et al., 2010

D. Majumdar, M.S. Pal, D. Majumdar

Effects of military load carriage on kinematics of gait

Ergonomics, 53 (2010), pp. 782-791

Orendurff et al., 2008

M.S. Orendurff, G.C. Bernatz, J.A. Schoen, G.K. Klute

Kinetic mechanisms to alter walking speed

Gait Posture, 27 (2008), pp. 603-610

Pal et al., 2009

M.S. Pal, D. Majumdar, M. Bhattacharyya, R. Kumar, D. Majumdar

Optimum load for carriage by soldiers at two walking speeds on level ground

Int. J. Ind. Ergon., 39 (2009), pp. 68-72

Pau et al., 2011

M. Pau, E. Saba, M. Pau

A study on the combined effect of obesity and load carriage on plantar pressure patterns of primary school children

Proc. Hum. Factors Ergon. Soc. Annu. Meet., 55 (2011), pp. 1606-1610

Perry, 1992 
J. Perry

Stride analysis

J. Perry (Ed.), Gait Analysis: Normal and Pathological Function, SLACK

Incorporated, Thorofare, NJ (1992), pp. 431-441

Piscoya et al., 2005

J.L. Piscoya, B. Fermor, V.B. Kraus, T.V. Stabler, F. Guilak

The influence of mechanical compression on the induction of osteoarthritisrelated biomarkers in articular cartilage explants

Osteoarthr. Cartil., 13 (2005), pp. 1092-1099

Rosenbaum et al., 1994

D. Rosenbaum, S. Hautmann, M. Gold, L. Claes

Effects of walking speed on plantar pressure patterns and hindfoot angular motion

Gait Posture, 2 (1994), pp. 191-197

Seeley et al., 2008

M.K. Seeley, B.R. Umberger, R. Shapiro

A test of the functional asymmetry hypothesis in walking

Gait Posture, 28 (2008), pp. 24-28

Segal, 2004

A. Segal, E. R, M. Orendurff, J. Shofer, M. O'Brien, B. Sangeorzan

The effect of walking speed on peak plantar pressure

Foot Ankle Int., 25 (2004), pp. 926-933

Simpson et al., 2011a

K.M. Simpson, B.J. Munro, J.R. Steele

Backpack load affects lower limb muscle activity patterns of female hikers during prolonged load carriage

J. Electromyogr. Kinesiol., 21 (2011), pp. 782-788

Simpson et al., 2011b 
K.M. Simpson, B.J. Munro, J.R. Steele

Effect of load mass on posture, heart rate and subjective responses of recreational female hikers to prolonged load carriage

Appl. Ergon., 42 (2011), pp. 403-410

Simpson et al., 2012

K.M. Simpson, B.J. Munro, J.R. Steele

Effects of prolonged load carriage on ground reaction forces, lower limb kinematics and spatio-temporal parameters in female recreational hikers

Ergonomics, 55 (2012), pp. 316-326

Singh and Koh, 2009

T. Singh, M. Koh

Effects of backpack load position on spatiotemporal parameters and trunk forward lean

Gait Posture, 29 (2009), pp. 49-53

Skaggs et al., 2006

D. Skaggs, S. Early, P. D'Ambra, V. Tolo, K. RM

Back pain and backpacks in school children

J. Pediatr. Orthop., 26 (2006), p. 6

Stevens, 2002

J. Stevens

Applied Multivariate Statistics for the Social Sciences

(fourth ed.)Lawrence Erlbaum Associates, Mahwah, NJ (2002)

Voloshin, 2000

A. Voloshin

The influence of walking speed on dynamic loading on the human musculoskeletal system

Med. Sci. Sports Exerc, 32 (2000), pp. 1156-1159 